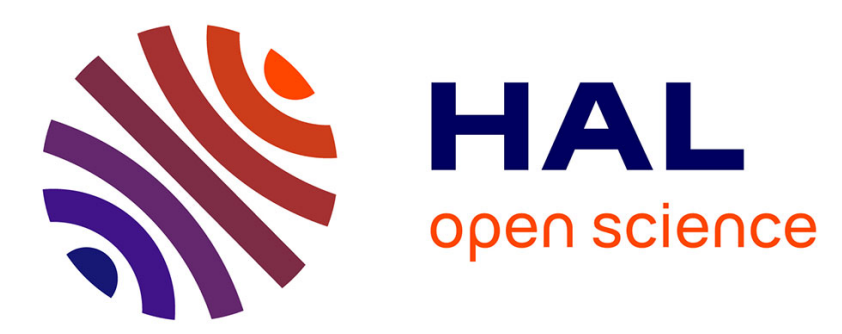

\title{
Raman-Brillouin light scattering in low-dimensional systems: Photoelastic model versus quantum model
}

Adnen Mlayah, Jean-Roch Huntzinger, Nicolas Large

\section{To cite this version:}

Adnen Mlayah, Jean-Roch Huntzinger, Nicolas Large. Raman-Brillouin light scattering in lowdimensional systems: Photoelastic model versus quantum model. Physical Review B: Condensed Matter and Materials Physics (1998-2015), 2007, 75 (24), pp.245303. 10.1103/PhysRevB.75.245303 . hal-00389556

\section{HAL Id: hal-00389556 https://hal.science/hal-00389556}

Submitted on 23 Apr 2018

HAL is a multi-disciplinary open access archive for the deposit and dissemination of scientific research documents, whether they are published or not. The documents may come from teaching and research institutions in France or abroad, or from public or private research centers.
L'archive ouverte pluridisciplinaire HAL, est destinée au dépôt et à la diffusion de documents scientifiques de niveau recherche, publiés ou non, émanant des établissements d'enseignement et de recherche français ou étrangers, des laboratoires publics ou privés. 


\title{
Raman-Brillouin light scattering in low-dimensional systems: Photoelastic model versus quantum model
}

\author{
Adnen Mlayah, Jean-Roch Huntzinger,* and Nicolas Large \\ Centre d'Elaboration des Matériaux et d'Etudes Structurales UPR 8011, CNRS-Université Paul Sabatier, \\ 29 rue Jeanne Marvig, Boîte Postale 94347, 31055 Toulouse Cedex, France
}

(Received 1 December 2006; revised manuscript received 20 April 2007; published 5 June 2007)

\begin{abstract}
Raman-Brillouin scattering by acoustic phonons in a free-standing nanosized silicon film is studied theoretically. Two well-known models are used to describe the inelastic light scattering. (i) The Raman-Brillouin quantum model in which the electronic states, and their interactions with light and sound, are taken into account explicitly. In this model, an effective Raman-Brillouin electronic density is introduced; it is very useful for analyzing experimental data when a large number of electronic states are involved as intermediate states in the light scattering process. Its spatial distribution depends on the optical excitation and electronic transitions of the system, and is directly connected to spectral features of the Raman-Brillouin scattering. In particular, diagonal and off-diagonal electronic densities are introduced in order to point out that diagonal electronvibration matrix elements are relevant for inelastic light scattering in nanosized objects, whereas only offdiagonal matrix elements are allowed for bulk materials. (ii) The photoelastic model is compared to the Raman-Brillouin quantum model and is discussed in terms of validity of the steplike profile of the photoelastic coefficient usually adopted in simulations of the Raman-Brillouin spectra of thin films and superlattices. It is shown that the use of trapezoidlike, rather than steplike, profiles of the photoelastic coefficient is more realistic.
\end{abstract}

DOI: 10.1103/PhysRevB.75.245303 PACS number(s): 73.22.Lp, 63.22.+m, 78.30.Er, 78.67.Bf

\section{INTRODUCTION}

Inelastic light scattering by acoustic vibrations has attracted much interest since the early work of Merz et al. ${ }^{1}$ and Colvard et $a .^{2}$ who observed light scattering by acoustic phonons due to Brillouin zone folding in semiconductor superlattices. Afterwards, many experimental and theoretical studies have extended the use of Raman-Brillouin scattering to the study of nanostructures ${ }^{3-5}$ consisting of spatially distributed quantum objects (wells, wires, dots, particles)..$^{3,4,6}$ The interest lies in the fact that the wavelengths of acoustic vibrations, in solids and in the $\mathrm{THz}$ frequency range, are of the order of few nanometers, i.e., well adapted to nanosized objects. ${ }^{7}$ Another important advantage of acoustic phonons is the delocalized nature of their displacement field. Unlike optic phonons, which are usually confined within one type of material, acoustic vibrations may extend over distances much larger than the average separation between distributed scatters. As a result, collective effects, due to spatial ordering, appear in the electron-phonon interaction and in the light scattering process. ${ }^{6,7}$ Hence, the information one can extract through modeling and simulations of the Raman-Brillouin spectra is very rich (size and shape distributions, spatial correlations,...).

Two main approaches are used for the interpretation and calculations of the acoustic phonon Raman-Brillouin scattering in nanostructures. First is the well-known Photoelastic model $^{8}$ (PEM) which assumes a modulation of the bulk material optical properties by acoustic vibrations through photoelastic coefficients, also known as Pockels coefficients. ${ }^{9}$ It was initially developed for the interpretation of Brillouin scattering in bulk materials. It has been then extended to two-dimensional structures ${ }^{4}$ (superlattices, cavities, membranes) and generalized recently by Lazarenkova et al. ${ }^{10}$ to three-dimensional ordered ensembles of self-assembled quantum dots. The PEM is very useful for studying acoustic cavity effects as shown by Giehler et al. ${ }^{11}$ and Fainstein et al. ${ }^{12}$ Acoustic confinement in semiconductor membranes (tens of nm thick) has been also pointed out recently by Sotomayor et al. ${ }^{13}$ using a detailed comparison between Raman-Brillouin measurements and the spectra simulated with the PEM. In the PEM only the acoustic vibrations are modified by reflections and transmissions at interfaces and surfaces; the electronic structure is taken into account only through the spatial variation of the photoelastic coefficients (which are material dependent). Optical cavity effects have been introduced in some cases. ${ }^{12-14}$ The PEM is valid for excitation energy far from any electronic transition (out of resonance). It is worthwhile to mention that the PEM is widely used also for the interpretation of the acoustic phonon echoes observed in time-resolved pump-probe experiments. ${ }^{15-18}$ The conclusions of the present work are mainly concerned with Raman-Brillouin scattering but they apply to the time modulation of the optical properties as well.

The second way of calculating the Raman-Brillouin efficiency is based on a quantum mechanical description of the light scattering. The latter is a three step process: incident photon absorption, phonon emission (or absorption), scattered photon emission. The electronic states play the role of intermediate states in the sense that the photon-phonon interaction is not direct, but occurs via electron-photon and electron-phonon interactions. Unlike in the PEM, in the Raman-Brillouin quantum model (RBQM) the electronic energies and wave functions are taken into account explicitly. This model has been successfully used for the simulation of resonantly excited Raman-Brillouin spectra, i.e., when the light scattering properties are determined by the electronic structure.

First studies of acoustic phonon scattering due to electronic wave-function localization were reported by Kop'ev et 
al. ${ }^{19}$ and Sapega et al. ${ }^{20}$ in GaAs/AlAs multiple quantum wells. In these structures, the electronic transitions were inhomogeneously broadened leading to resonant selection of localized electron and hole wave functions of individual quantum wells. Because of the spatial localization of the intermediate electronic states the wave vector exchanged during the scattering process is no longer transferred to a unique vibration mode but to all modes of the Brillouin zone. As a consequence, the spectral shape of the acoustic phonon Raman-Brillouin scattering reflects the spatial distribution of the excited electronic density. ${ }^{6,7}$ The RBQM has been used to extract characteristic features of the electronic states such as localization and correlation lengths. ${ }^{21}$ These basic ideas have been recently extended to semiconductor quantum dots to include three-dimensional confinement effects ${ }^{22,23}$ and spatial ordering effects. ${ }^{24-30}$

Despite the fact that the PEM and RBQM were extensively used for the interpretation of the Raman-Brillouin measurements, the connection between both models still needs to be clarified. This is particularly important for the interpretation of experiments performed close to resonance, for which the validity of the PEM becomes questionable. On the other hand, under resonant excitation, only a few intermediate electronic states are responsible for the light scattering and are therefore taken into account in the RBQM. Out of (or close to) resonance, this approximation is no longer valid and the whole density of electronic states should be included in the RBQM. This is also the case when the energy separation between electronic states is smaller than the homogeneous broadening of the resonant optical transitions.

In this work we present calculations of Raman-Brillouin scattering using both the PEM and RBQM. In order to keep within analytical and tractable calculations, the results are presented for a simple model system consisting of a freestanding silicon film. They can, however, be easily generalized to semiconductor multi layers and to embedded quantum wires or dots. The main points addressed in this work are (i) the dependence of the Raman-Brillouin spectra simulated with the RBQM on the optical excitation energy. Unlike in most of the published works, a large number of confined electronic states are here taken into account, thus leading to strong interference effects between different scattering paths. In particular, the effective Raman-Brillouin electronic density (RBED), ${ }^{31}$ introduced by some of us in the case of quantum dots, is shown to be very useful for analyzing light scattering in layered structures. (ii) The comparison between the photoelastic model and the Raman-Brillouin quantum model. It is well known that the photoelastic constants strongly depend on energy. ${ }^{32}$ They change sign and acquire an imaginary part near band gaps. The spatial distribution of the RBED depends on the optical excitation energy. This property is used to discuss the validity of the PEM for optical excitation close to resonance with confined electronic transitions. (iii) A steplike profile of the photoelastic constant is usually assumed for the simulation of the Raman-Brillouin spectra when using the PEM. Is this simple profile realistic and can it still be used for thin layers in which quantum effects can no longer be ignored? In this work we show that the RBED is a tool well adapted for the discussion of this still open question. In particular, an alternative to the widely used steplike profile of the photoelastic coefficient is proposed for very thin layers.

\section{MODELS}

\section{A. Raman-Brillouin quantum model}

In this section we briefly recall the RBQM and introduce the effective Raman-Brillouin electronic density for a free standing quantum film. The quantum efficiency of RamanBrillouin scattering can be obtained from perturbation theory. Whereas the first and second order correction terms describe, respectively, the optical absorption and Rayleigh scattering, the third order term expresses the inelastic light scattering. When mediated by conduction states, its efficiency is proportional to ${ }^{5,6,20}$

$$
\left|\sum_{e, e^{\prime}, h} \frac{\left\langle h\left|H_{e-\mathrm{pht}}^{s}\right| e^{\prime}\right\rangle\left\langle e^{\prime}\left|H_{e-\mathrm{phn}}\right| e\right\rangle\left\langle e\left|H_{e \text {-pht }}^{i}\right| h\right\rangle}{\left(E_{s}-E_{e^{\prime}-h}+i \gamma_{e^{\prime}-h}\right)\left(E_{i}-E_{e-h}+i \gamma_{e-h}\right)}\right|^{2},
$$

where $e, e^{\prime}$, and $h$ are electron and hole eigenstates; $E_{e-h}$ and $\gamma_{e-h}$ are the energy and the homogeneous linewidth of the $e-h$ transition, respectively; $E_{e-h}$ is defined as $E_{e-h}=E_{0}+E_{e}+E_{h}$, where $E_{0}$ is the bulk direct band gap and $E_{e}$ (respectively, $E_{h}$ ) is the electron (respectively, hole) confinement energy. $E_{i}$ and $E_{s}=E_{i} \pm \hbar \omega_{\text {phn }}$ are, respectively, the incident and scattered photon energies $\left(\hbar \omega_{\text {phn }}\right.$ being the energy of the absorbed or emitted phonon). $H_{e \text {-pht }}$ and $H_{e \text {-phn }}$ are the electronphoton and electron-phonon interaction Hamiltonians, respectively.

The sum in Eq. (1) runs over all intermediate conduction states $e$ and $e^{\prime}$ and initial valence states $h$ which can be described by Bloch wave functions $\psi_{e(h)}(\mathbf{r})=u_{c(v)}(\mathbf{r}) \phi(\mathbf{r})$; $u_{c(v)}(\mathbf{r})$ being the atomiclike wave function and $\phi(\mathbf{r})$ the slowly varying envelope functions.

In the following, we will restrict the discussion to twodimensional quantum films $\left(L_{z} \ll L_{x}, L_{y}\right)$. This because we would like to focus on the effects of wavefunction localization and mixing (size effects) on the Raman-Brillouin scattering rather than on dimensionality and shape effects. Moreover, experimental results are available for silicon membranes. ${ }^{13}$

For the sake of simplicity, we shall consider only zero in-plane wave vector electron-hole transitions. This restriction allows to discuss the relation between PEM and RBQM with simplified notations and without loss of generality. It must be, however, reconsidered for a detailed comparison with experiments.

Assuming that electrons and holes are perfectly confined within the film, the envelope wave functions are then given by

$$
\phi_{n}(z)=\sqrt{\frac{2}{L_{z}}} \sin \left(\frac{n \pi z}{L_{z}}\right),
$$

where $L_{z}$ is the film thickness. Using the parabolic band approximation, the electron and hole confinement energies are, respectively, $E_{e}^{n}=\frac{\hbar^{2} \pi^{2}}{2 m_{e}} \frac{n^{2}}{L_{z}^{2}}$ and $E_{h}^{n}=\frac{\hbar^{2} \pi^{2}}{2 m_{h}} \frac{n^{2}}{L_{2}^{2}}$, where $n$ is an integer; $m_{e}=0.28 m_{0}$ (Ref. 34) and $m_{h}=0.49 m_{0}$ (Ref. 35) are, respectively, the electron and hole effective masses used in the 
calculations. Moreover, for the bulk band gap we used $E_{0}$ $=4.2 \mathrm{eV}$ which corresponds to direct transitions from the top of the valence band to the $\Gamma_{2}$ conduction band minimum. ${ }^{36,37}$ For this transition the parabolic band approximation is valid at least around the $\Gamma_{2}$ point. Obviously, the confinement energies of electrons and holes in very thin silicon layers are overestimated by the parabolic band approximation. More sophisticated band structure calculations ${ }^{36}$ are needed but this is out of the scope of the present work.

The electron-photon interaction Hamiltonian is given by $H_{e-\mathrm{pht}}^{i(s)}=\frac{q \vec{p} \cdot \vec{A}_{i(s)}}{m_{e}}$ as usual; $\vec{p}$ being the electron quantum momentum and $A_{i(s)}$ the vector potential of the incident (scattered) radiations. We assume deformation-potential (DP) electronphonon interaction involving longitudinal acoustic (LA) vibrations. Thus, the electron-phonon Hamiltonian, acting on the envelope wave functions, reads $H_{e-\mathrm{phn}}=D_{c(v)} \vec{\nabla} \cdot \vec{u}$, where $\vec{u}$ is the displacement vector, and $D_{c(v)}$ is the conduction (valence) band deformation potential energy. Using stress free boundary conditions at the film surfaces, the displacement field of confined LA vibrations along the $z$ direction is given by

$$
u_{m}(z)=\sqrt{\frac{\hbar}{2 \rho V \omega_{m}}} \cos \left(\frac{m \pi z}{L_{z}}\right),
$$

where $\rho$ and $V$ are the mass density and film volume, respectively; the frequency of LA phonons, assuming a linear dispersion, is proportional to the longitudinal sound velocity $v_{L}$ and the phonon wave vector $k_{m}=\pi \frac{m}{L_{z}}: \omega_{\mathrm{phn}}=\omega_{m}=v_{L} k_{m}(m$ is an integer).

When only one intermediate electronic state is resonantly excited by the probe light, the sum of scattering amplitudes [Eq. (1)] can be limited to one term; the off-resonance terms can be neglected. In that case the Raman-Brillouin peak positions and intensities are directly related to the spatial distribution of the electronic density selected by the optical excitation. Otherwise, one has to sum a large number of terms which leads to strong interference effects in the overall scattering efficiency. Therefore, the characteristics of the RamanBrillouin spectra can hardly be related to characteristic features of the excited electronic states.

In order to overcome this difficulty, we proposed to rewrite the scattering efficiency [Eq. (1)] by introducing a Raman-Brillouin electronic density defined as ${ }^{31}$

$$
\rho_{\mathrm{RB}}\left(E_{i}, E_{s}, z\right)=\frac{1}{R\left(E_{i}, E_{s}\right)} \sum_{e, e^{\prime}, h} R_{h, e^{\prime}}^{s}\left(E_{s}\right) \phi_{e^{\prime}}^{*}(z) R_{e, h}^{i}\left(E_{i}\right) \phi_{e}(z),
$$

where $R_{e, h}^{i(s)}\left(E_{i(s)}\right)$ is a dimensionless resonance factor given by

$$
R_{e, h}^{i(s)}(E)=\frac{\left\langle e\left|H_{e-\mathrm{pht}}^{i(s)}\right| h\right\rangle}{E_{i(s)}-E_{e-h}+i \gamma_{e-h}}
$$

and

$$
R\left(E_{i}, E_{s}\right)=\sum_{e, h} R_{h, e}^{s}\left(E_{s}\right) R_{e, h}^{i}\left(E_{i}\right)
$$

is a normalization factor satisfying $\int \rho_{\mathrm{RB}}(z) d z=1$.

$\rho_{\mathrm{RB}}(z)$ is determined by the sum over the initial hole states of the overlapping between the effective electronic state $\Sigma_{e} R_{e, h}^{i}\left(E_{i}\right) \phi_{e}(z)$ excited at the probe laser energy $E_{i}$, and the effective electronic state $\Sigma_{e^{\prime}} R_{h, e^{\prime}}^{s}\left(E_{s}\right) \phi_{e^{\prime}}(z)$ giving rise to emission of a scattered photon at energy $E_{s}=E_{i} \pm \hbar \omega_{\text {phn }}$; $\rho_{\mathrm{RB}}(z)$ has real and imaginary contributions because of the homogeneous broadening of the electron-hole transitions [Eq. (5)]. Using Eq. (4), Eq. (1) becomes

$$
\left|R\left(E_{i}, E_{s}\right) D_{c} \int \rho_{\mathrm{RB}}(z) \frac{\partial u_{m}(z)}{\partial z} d z\right|^{2} .
$$

From Eq. (7), one can see that $\rho_{\mathrm{RB}}(z)$ is the electronic density distribution that interacts with the phonon field and is responsible for the Raman-Brillouin scattering.

Strictly speaking, Eq. (7) does not contain new physics with respect to Eq. (1) in the sense that both equations are equivalent. However, using Eq. (7), one is still able to plot an electronic density distribution which is directly connected to characteristic features of the Raman-Brillouin spectra, although many electronic states are involved in the light scattering. Moreover, as we will show, the RBED is the discussion thread between the Raman-Brillouin quantum model and the photoelastic model.

\section{B. Photoelastic model}

In the photoelastic model, the light scattering is due to the modulation of the dielectric susceptibility by the vibration modes. Here, we discuss the integral form of the photoelastic model as introduced in Ref. 4 and used by many authors for the interpretation of Raman-Brillouin scattering in lowdimensional systems. Notice that the photoelastic model was initially develop in a differential equation form. ${ }^{33}$ However, both forms (integral and differential) assume a steplike photoelastic constant and this is the main point to be discussed in the following sections.

For scattering by longitudinal acoustic modes the RamanBrillouin efficiency is proportional to $\mathrm{t}^{4,12,14}$

$$
\left|\int \vec{A}_{s}^{*}(z) \vec{A}_{i}(z) P(z) \frac{\partial u_{m}(z)}{\partial z} d z\right|^{2},
$$

where $P(z)$ is the spatial variation of the photoelastic coefficient, and $\vec{A}_{i}\left(\vec{A}_{s}\right)$ is the incident (scattered) photon field. Optical and acoustical cavity effects are introduced through wave reflections and transmissions at the interfaces. ${ }^{11,12,14}$

In order to introduce the spatial variation of the incident and scattered photon fields in a simple way, we assume (for both PEM and RBQM) $A_{i(s)}(z)$ of the form $\cos \left[k_{i(s)}(z\right.$ $\left.\left.-L_{z} / 2\right)\right] ; k_{i(s)}$ being the incident or scattered photon wave vector component along the $z$ direction. The in-plane component of the incident and scattered wave vectors are neglected in the backscattering configuration (excitation and detection along the $z$ direction). Optical cavity effects, due to reflections at the material/air interfaces, may play an impor- 
tant role because the symmetry of the electromagnetic fields inside the layer is responsible for a selection of the confined vibration modes that gives rise to Raman-Brillouin scattering. Although, important for the interpretation of experimental data such effects are not addressed in this paper. Moreover, with the form adopted for $A_{i(s)}(z)$ (standing waves) the backward and forward scattering configurations are equivalent.

For a bulk material, the photoelastic coefficient is a constant within the material volume (of which dimensions are much larger than the wavelength of photons and phonons). In that case the integral in Eq. (8) reduces to a delta function and the wave vector conservation $k_{i}-k_{s}=k_{m}$ is fulfilled. Hence, the spectrum of the scattered light consists of a single line, namely, the Brillouin peak.

In superlattices, $P(z)$ is a periodic steplike function along the growth direction. According to Eq. (8), the wave vector conservation is fulfilled at integer numbers of the superlattice wave vector leading to several peaks in the Raman-Brillouin spectrum. It is well known that from the frequencies of these peaks, one is able to extract the superlattice period. ${ }^{1,2,4}$ The intensities ratios of the peaks are directly related to the photoelastic coefficients of the layered materials.

For our model system, consisting of a single twodimensional layer, $P(z)$ is a rectangular function. The photoelastic coefficient being constant inside the layer and zero outside. In the following we will refer to this $P(z)$ profile as a "steplike" profile. In that case, the wave vector conservation law breaks down (along the $z$ direction) and all acoustic phonon modes become allowed. Their Raman activity depend on their symmetry (with respect to the middle of the layer) and on the electromagnetic fields inside the layer [see Eq. (8)].

\section{RESULTS AND DISCUSSIONS}

\section{A. Construction of the Raman-Brillouin electronic density}

Figure 1 shows the spatial distribution of the RBED calculated using Eq. (4) for a film thickness $L_{z}=10 \mathrm{~nm}$. Since we are interested in studying the change of the RamanBrillouin scattering around the fundamental electronic transition, $\rho_{\mathrm{RB}}(z)$ was generated using the first fifteen electron and first fifteen hole states. A homogeneous broadening $\gamma_{e-h}=25 \mathrm{meV}$ (thermal broadening at room temperature) was used for all transitions. The RBED shown in Fig. 1 was calculated for various values of the detuning between the incident photon energy and the fundamental electron-hole transition; the detuning is defined as $\delta=E_{i}-\left(E_{0}+E_{e}^{1}+E_{h}^{1}\right)$ in which $E_{e}^{1}\left(E_{h}^{1}\right)$ is the confinement energy of the fundamental electron (hole) state. We also define the reduced detuning $\delta^{\prime}=\delta /\left(E_{e}^{1}+E_{h}^{1}\right)$ and reduced broadening $\gamma^{\prime}=\gamma_{e-h} /\left(E_{e}^{1}+E_{h}^{1}\right)$. The scattered photon energy is fixed at the Stokes value $E_{s}$ $=E_{i}-\hbar \omega_{1}$ corresponding to emission of the first confined acoustic vibration mode.

Because $\hbar \omega_{\text {phn }} \ll \gamma_{e-h}$, in-coming and out-going resonances occur simultaneously, and the RBED is rather independent of the acoustic vibration energy (this point will be reconsidered later on). For excitation well below the funda-

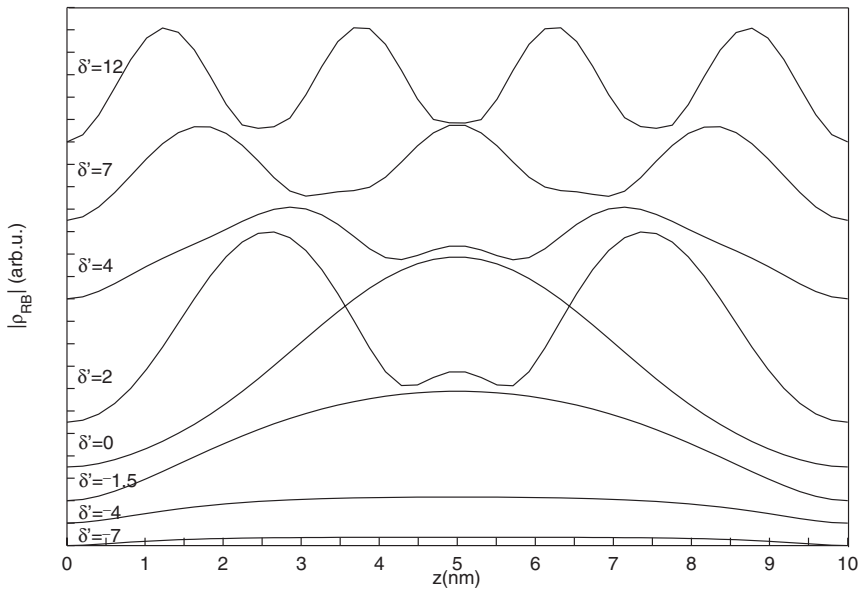

FIG. 1. Modulus of the Raman-Brillouin electronic density along the $z$ axis of a 10-nm-thick layer, and for reduced detuning ranging from $\delta^{\prime}=-7$ to $\delta^{\prime}=12$. The reduced homogeneous broadening is $\gamma^{\prime}=\gamma_{e-h} /\left(E_{e}^{1}+E_{h}^{1}\right)=1.2$. The plots were shifted vertically for clarity.

mental electron-hole transition $\left(\delta^{\prime}=-7\right.$ and -4$)$, the RBED distribution within the layer is quasiuniform (i.e., constant except in the vicinity of the film surfaces). In this situation (far from resonance), there is no selection of a particular transition. Close to resonance $\left(\delta^{\prime}=-1.5\right)$, the contribution of the first confined electron-hole transition emerges and becomes dominant for resonant excitation $\left(\delta^{\prime}=0\right)$. Higher energy confined transitions come into resonance, for $\delta^{\prime}=2,4$, 7 , and 12, and give rise to strong oscillations of the RBED.

Figure 2 shows resonance profiles calculated according to Eq. (6) for three values of the reduced homogeneous broadening $\gamma^{\prime}=\gamma_{e-h} /\left(E_{e}^{1}+E_{h}^{1}\right)$. For rather small homogeneous broadening $\left(\gamma^{\prime}=0.12\right.$ and 1.2) resonance peaks occur at each confined electron-hole transition: the selection of a given transition by the optical excitation is very efficient and the

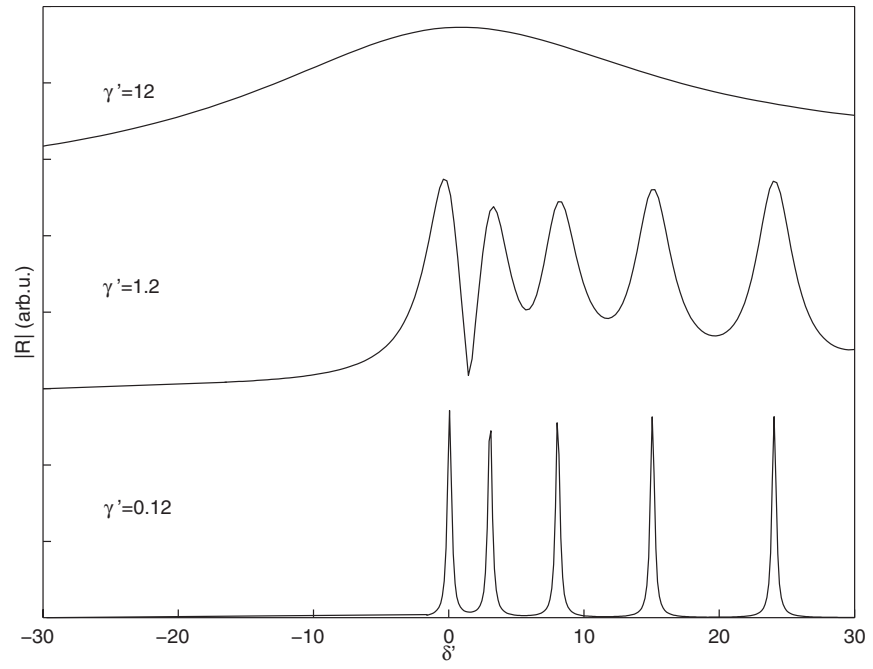

FIG. 2. Raman-Brillouin intensity as a function of the detuning $\delta^{\prime}$ for a 10-nm-thick layer. Resonance profiles are shown for homogeneous broadening $\gamma=2.5 \mathrm{meV}\left(\gamma^{\prime}=0.12\right), 25 \mathrm{meV}\left(\gamma^{\prime}=1.2\right)$, and $250 \mathrm{meV}\left(\gamma^{\prime}=12\right)$. 


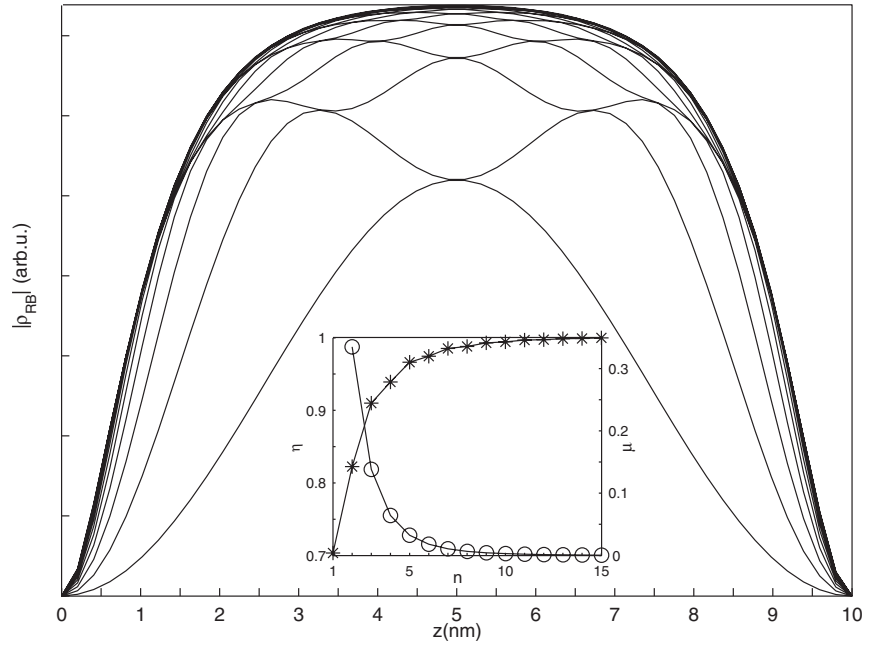

FIG. 3. Construction of the RBED for $\delta^{\prime}$ $=-7$ (excitation below the fundamental optical resonance) and $\gamma^{\prime}$ $=1.2$. $n$ is the number of electron and the number of hole states used in the calculations. Starting from the lowest plot $n=1$ (one electron and one hole states), $n$ is increased up to 15 . The inset shows convergence curves defined as $\mu=\| \rho_{\mathrm{RB}, \max }^{n}|-| \rho_{\mathrm{RB}, \max }^{n-1}||\left|\rho_{\mathrm{RB}, \max }^{n}\right|$ (circles) and $\eta=1-|| \rho_{\mathrm{RB}, \max }^{15}|-| \rho_{\mathrm{RB}, \max }^{n}|/| \rho_{\mathrm{RB}, \max }^{15} \mid$ (stars).

light scattering is mediated by the corresponding electron and hole states. For larger homogeneous broadening $\left(\gamma^{\prime}\right.$ $=12$ ), many electron-hole transitions are excited leading to interferences between different scattering paths: the lowenergy tail (negative detuning in Fig. 2) is due to the resonance effect and also to the fact that, for negative detuning, all scattering amplitudes have nearly the same phase (that of the optical excitation). In that case, the interferences between the different scattering amplitudes (terms of sum 4) are constructive. Whereas, for positive and large detuning $\left(\delta^{\prime} \gg 1\right)$ some scattering amplitudes have a positive phase and others a negative phase depending on whether the energy of the excited (and detected) electron-hole transition is larger or smaller than the excitation energy. As a consequence, the scattered intensity falls down for positive detuning due to destructive interference between all scattering amplitudes.

Figure 3 shows the construction of the Raman-Brillouin electronic density for $\delta^{\prime}=-7$ and $\gamma^{\prime}=1.2$ (plotted in Fig. 1). The number $n$ for electron and hole states is increased from 1 to 15 .

One can see that even for excitation well below $\left(\delta^{\prime}\right.$ $=-7$ ) the fundamental electron-hole transition, high energy transitions play an important role in the construction of the RBED (i.e., in the scattering process). For instance, the maximum value of the RBED reaches only $91 \%$ of its final value (inset of Fig. 3) when including three electron and three hole states. The convergence curves and the plots in Fig. 3 show that the RBED tend to be quasiuniform and does not evolve once ten electron and ten hole states are taken into account.

As can be noticed, the convergence of the RBED is very rapid. This allows generating Raman-Brillouin spectra, within a reasonable computation time, even for large systems.

From Eq. (4) it is evident that the RBED has a real part and an imaginary part; the latter is due to the homogeneous
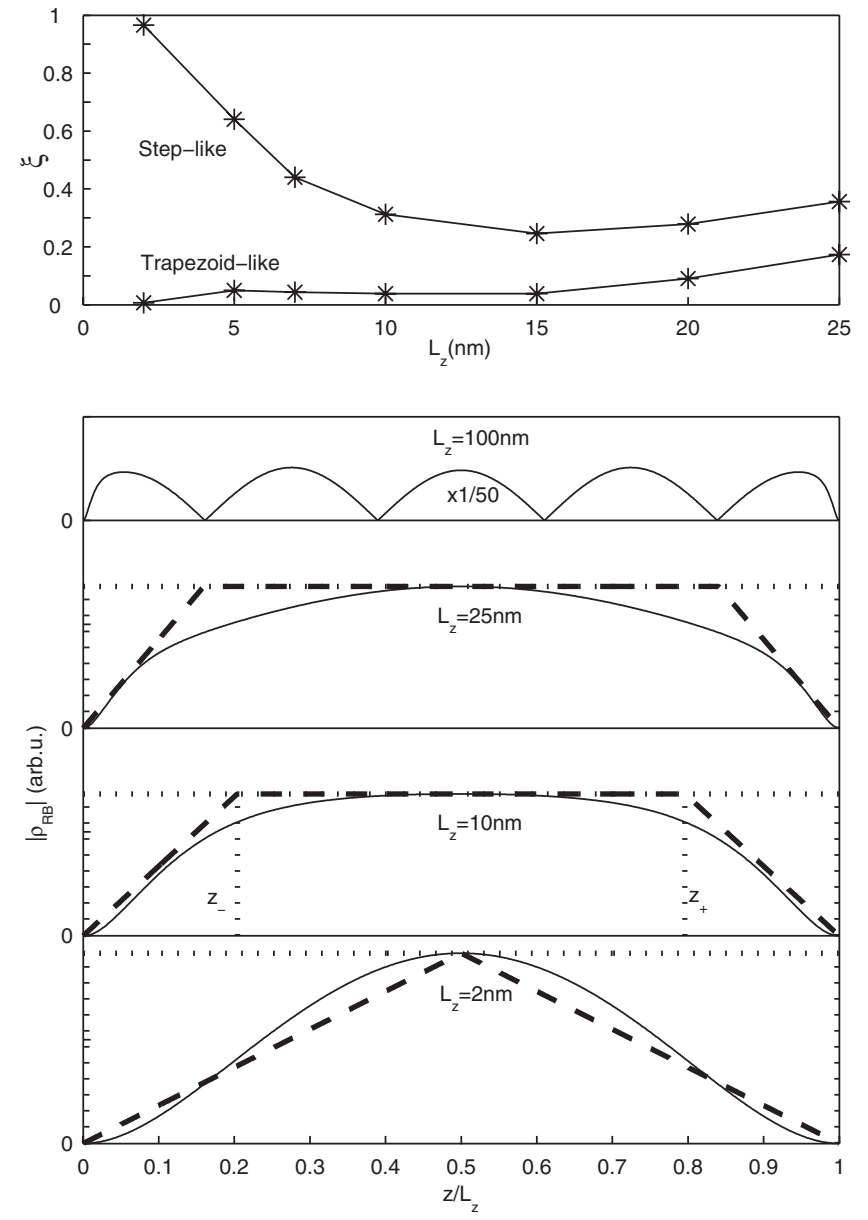

FIG. 4. Lower panel: Modulus of the RBED along the $z$ axis for layer thickness ranging from $L_{z}=2$ to $100 \mathrm{~nm}$. The excitation energy is fixed at $E_{i}=4.075 \mathrm{eV}$ and the homogeneous broadening is $\gamma=25 \mathrm{meV}$. Reduced detuning and homogeneous broadening $\left(\delta^{\prime}, \gamma^{\prime}\right)$ are, from bottom to top, $(-1.2,0.05),(-7,1.2),(-38,7.4)$, and $(-590,120)$. For each $L_{z}$ are shown the steplike profile (bold dotted line) and the trapezoidlike profile (bold dashed line); $z_{-}$and $z_{+}$are the $z$ coordinates (dashed lines) which define the trapezoid. Upper panel: deviation of the steplike and trapezoidlike profiles from the RBED evaluated as $\xi_{\text {step(trap) }}=\mid S_{\text {step(trap) }}$ $-\int\left|\rho_{\mathrm{RB}}(z)\right| d z\left|/ \int\right| \rho_{\mathrm{RB}}(z) \mid d z$.

broadening of the electronic transitions. For $\delta^{\prime}=-7$ and $\gamma^{\prime}$ $=1.2$ the imaginary part of the RBED is rather small in comparison with the real part. It, however, increases when approaching the resonance and has a noticeable influence on the spatial distribution of $\left|\rho_{\mathrm{RB}}(z)\right|$.

\section{B. Size dependence of the RBED}

The lower panel of Fig. 4 plots the distribution of the RBED along the $z$ axis for various layer thicknesses $L_{z}$. The excitation energy $E_{i}$ and homogeneous broadening $\gamma$ are fixed at $4.075 \mathrm{eV}$ and $25 \mathrm{meV}$, respectively. In that case, the reduced detuning $\delta^{\prime}$ and homogeneous broadening $\gamma^{\prime}$ depend on the layer thickness as indicated in the figure caption. For very thin layers $\left(L_{z}=2 \mathrm{~nm}\right)$ both $\delta^{\prime}$ and $\gamma^{\prime}$ are small. Then, the fundamental electron-hole transition is efficiently 


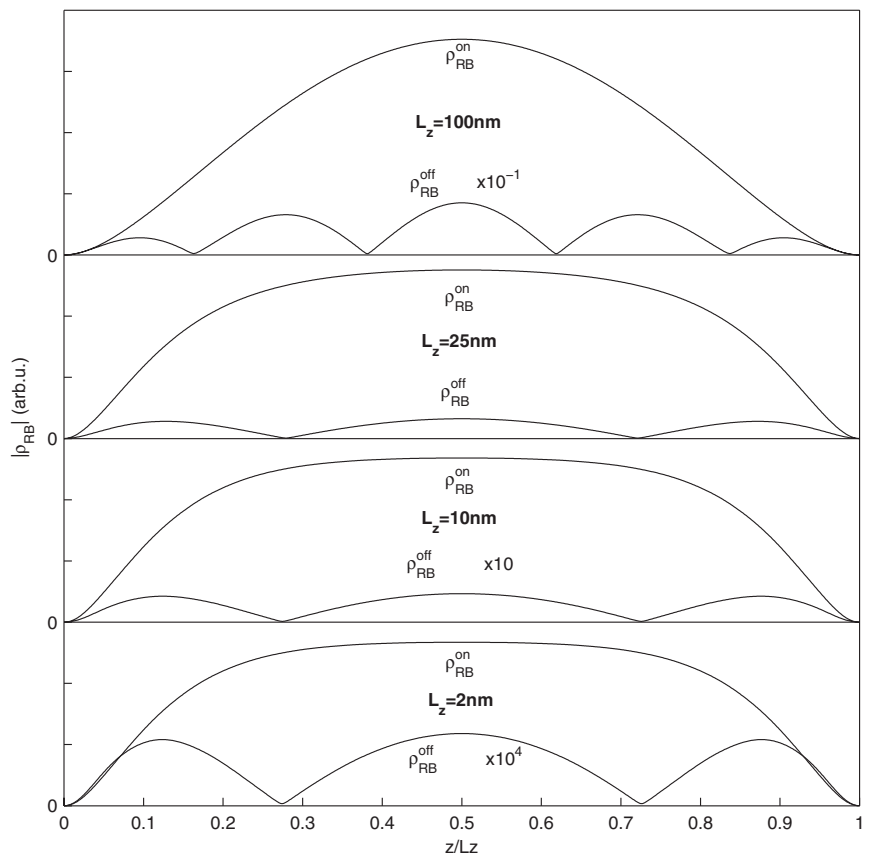

FIG. 5. Diagonal $\left(\rho_{\mathrm{RB}}^{\text {on }}\right)$ and off-diagonal $\left(\rho_{\mathrm{RB}}^{\text {off }}\right)$ RBED modulus along the $z$ axis for $L_{z}=2,10,25$, and $100 \mathrm{~nm}$; detuning and homogeneous broadening are respectively $\delta^{\prime}=-7$ and $\gamma^{\prime}=1.2$.

selected by the optical excitation. For this reason the RBED reduces to the density distribution associated with the first confined electron and hole states [Eq. (2)]. With increasing layer thickness, the energy separation between the confined electronic states decreases and many transitions come into resonance. This leads to a quasiuniform distribution of the RBED for $L_{z}=10$ and $25 \mathrm{~nm}$ as shown in Figs. 3 and 4 . However, for $L_{z}=100 \mathrm{~nm}$ the RBED oscillates and strongly deviates from a quasiuniform distribution. In fact, it is the wave vector conservation law that starts to come out with increasing layer thickness.

Indeed, for $k_{i(s)} L_{z} \ll 1$, i.e., for a layer thickness much smaller than the (incident and scattered) photon wavelength (around $150 \mathrm{~nm}$ inside the layer) the electromagnetic fields $A_{i}(z)$ and $A_{s}(z)$ are slowly varying functions along the $z$ axis. Therefore, the electron-photon matrix elements are proportional to the overlapping between the electron and hole wave functions. Thus, only electron and hole states having the same envelope wave functions are relevant for both the photon absorption and emission steps. As a consequence, the intermediate states $e$ and $e^{\prime}$ in Eqs. (1) and (7) are inevitably the same and the RBED is composed of diagonal matrix elements. The RBED plotted in Figs. 1 and 3 are mainly due to such diagonal contributions $\left(k_{i(s)} L_{z} \approx 0.1\right)$. Off-diagonal transitions have been taken into account but are negligible for $L_{z} \lesssim 10 \mathrm{~nm}$.

Figure 5 shows the spatial distribution of both diagonal and off-diagonal Raman-Brillouin electronic densities. For these plots $E_{i}$ and $\gamma$ are varied so that the reduced detuning and homogeneous broadening are fixed $\left(\delta^{\prime}=-7\right.$ and $\left.\gamma^{\prime}=1.2\right)$. This allows one to compare similar resonance conditions for the different layer thicknesses.

With increasing layer thickness the off-diagonal part of the RBED increases and becomes important for $L_{z}=100 \mathrm{~nm}$
(Fig. 5). Indeed, for a layer thickness comparable to the absorbed and emitted photon wavelengths $k_{i(s)} L_{z} \sim 1$, the spatial variation of the electromagnetic fields allows transitions between electron and hole states having different envelope wave functions. Therefore, different states $e$ and $e^{\prime}$ in Eqs. (1) and (7) can be involved in the light scattering. In the limit of $k_{i(s)} L_{z} \gg 1$ the RBED involves only transitions that fulfill the wave vector conservation rule at the electron-photon interaction steps (emission and absorption). In that case states $e$ and $e^{\prime}$ differ by the exchanged wave vector.

It is interesting to notice that the RBED shown in Fig. 5 for $L_{z}=2 \mathrm{~nm}$ strongly differs from the one plotted in Fig. 4 for the same layer thickness. The reduced detuning is not the same in these figures. In Fig. 5 the optical excitation is well below $\left(\delta^{\prime}=-7\right)$ the electron-hole transition. It is much larger, in absolute value, than the separation between the confined energy levels. In that case, the resonance factors associated with the different transitions are similar. That's why the RBED tends to be quasiuniform for a very negative detuning.

The study of the RBED distribution shows that the optical transitions giving rise to the light scattering process strongly depends on size effects. This is particularly important for the emission and absorption of vibration modes and for the interpretation of experimental data.

In the photoelastic model a steplike photoelastic coefficient (constant within the layer and zero outside) is usually assumed. By comparing Eqs. (7) and (8) one can see that the product $A_{s}^{*}(z) A_{i}(z) P(z)$ identifies with the RBED $\rho_{\mathrm{RB}}(z)$. For $k_{i(s)} L_{z} \ll 1$ (i.e., nanosized layer thickness), $A_{s}^{*}(z) A_{i}(z)$ is rather constant and thus it is mainly the photoelastic coefficient profile that determines the Raman-Brillouin spectra in the photoelastic model. That's why, in the following, we compare directly $\rho_{\mathrm{RB}}(z)$ and $P(z)$.

First, as can be seen in Fig. 4, the RBED is far from being constant within the layer; it vanishes at the film surfaces because only the first confined transitions significantly contribute to the RBED (Fig. 3). In other words, if all transitions could equally contribute to the light scattering, the RBED would approach a steplike profile. This is, of course, not the case since high energy transitions are far away from the optical excitation, thus leading to very small resonance factors.

The top panel of Fig. 4 presents a comparison between the RBED distribution of Fig. 4, the steplike profile usually adopted for the photoelastic coefficient and a proposed trapezoidlike profile. This comparison is performed for fixed excitation energy $(4.075 \mathrm{eV})$ and homogeneous broadening $(25 \mathrm{meV})$. The parameter used to evaluate the deviation of the RBED from the steplike or from the trapezoidlike profile is defined as $\xi=\left|S_{\text {step(trap) }}-\int\right| \rho_{\mathrm{RB}}(z)|d z| / \int\left|\rho_{\mathrm{RB}}(z)\right| d z$, where $S_{\text {step(trap) }}$ is the integral of the steplike (trapezoidlike) profile over the layer thickness. The amplitude of the steplike and trapezoidlike profiles are fixed to the maximum value of the RBED inside the layer (see Fig. 4).

It is clear that the steplike profile shows strong deviations from the RBED for very narrow layers (less than $10 \mathrm{~nm}$ ). The trapezoidlike profile fits much better the RBED distribution. In other words, by forcing the photoelastic coefficient to vanish at the layer surfaces, the quantum nature of the film 
is, in that artificial way, taken into account (the thin layer is no longer considered simply as a part of a bulk material from the view point of the optical properties). For $L_{z} \geqslant 30 \mathrm{~nm}$ the RBED starts to oscillate (see plot for $L_{z}=100 \mathrm{~nm}$ in Fig. 4). It strongly deviates from the steplike and trapezoidlike profiles because the spatial variation of $A_{s}^{*}(z) A_{i}(z)$ is not taken into account when only the photoelastic coefficient is plotted.

Second, by studying the variation of the RBED as a function of $L_{z}$ we are able to propose trapezoidlike profiles that fit the RBED for layer thicknesses ranging from 2 to $25 \mathrm{~nm}$; the parameters $z_{-}$and $z_{+}$defined in Fig. 4 are $z_{-}=0.74$ $+0.13 L_{z}$ and $z_{+}=-0.74+0.87 L_{z}$, where $L_{z}$ is in nm. Notice that for very thin layers $\left(L_{z}=2 \mathrm{~nm}\right.$ in Fig. 4$)$ the trapezoidlike profile becomes a trianglelike profile and obviously it strongly deviates from a steplike profile. By comparing the RBED distribution with these profiles it clearly appears that, for layer thickness ranging from 2 to $25 \mathrm{~nm}$, the proposed trapezoidlike profile is more realistic than the steplike profile usually assumed for the photoelastic coefficient. For larger layer thickness $\left(L_{z} \geqslant 25 \mathrm{~nm}\right)$ the deviations of the steplike and trapezoidlike profiles from the RBED indicate that the $A_{s}^{*} A_{i}$ terms should be taken into account, even for $L_{z}$ about five times smaller than the photon wavelengths.

Finally, strong deviations of the RBED distribution from the steplike and trapezoidlike photoelastic profiles are evident if one consider resonant excitation (see Fig. 1): both profiles are unable to approximate the RBED for excitation close to and above the fundamental electron-hole transition. The PEM, in which a real and dispersionless PE coefficient is assumed, is valid only for excitation below the fundamental electron-hole transition. That is why we compared the RBQM and PEM for negative $\delta^{\prime}$ (in Fig. 4). We now investigate the emission and absorption of acoustic vibrations and compare the inelastic light scattering spectra simulated using the RBED (quantum model) and the photoelastic model in which either the steplike or trapezoidlike profile of the of the photoelastic constant is assumed.

\section{Simulations of Raman-Brillouin spectra}

\section{Diagonal/off diagonal contributions}

Figure 6 presents Raman-Brillouin spectra generated using Eq. (7). The peaks in the Stokes and anti-Stokes regions are due to emission and absorption of confined acoustic vibrations (their frequencies scale as the inverse of the layer thickness $L_{z}$ ). For each $L_{z}$ are plotted the spectra simulated with either diagonal or off-diagonal RBED (shown in Fig. 5). One must, however, keep in mind that the overall scattered intensity is the coherent sum of the diagonal and off-diagonal contributions.

As mentioned above, for $L_{z}=2,10$, and $25 \mathrm{~nm}$ the Raman-Brillouin spectra are determined by the diagonal part of the RBED, mainly. Off-diagonal RBED contributes only a little to the light scattering. It also means that, for $k_{i(s)} L_{z}$ $\ll 1$, mainly diagonal electron-vibration matrix elements are responsible for the inelastic light scattering. This can be deduced directly from Eq. (1) by letting the intermediate states $e$ and $e^{\prime}$ be the same. With increasing layer thickness offdiagonal electron-vibration matrix elements come out since

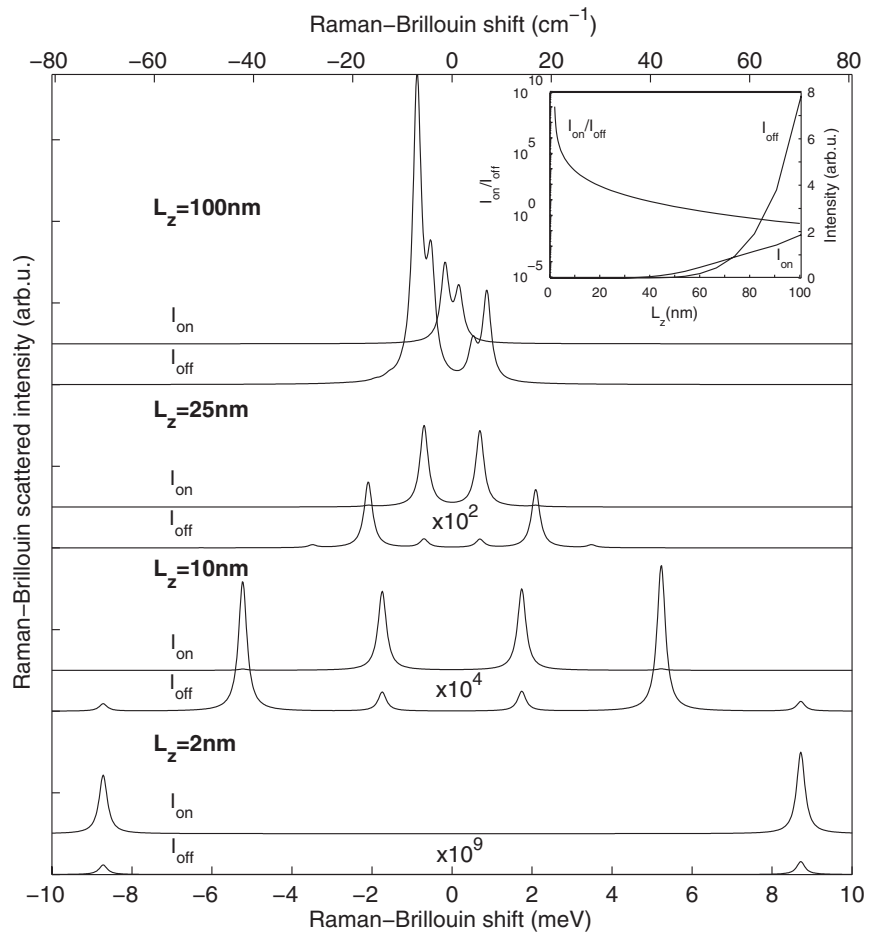

FIG. 6. Light scattering spectra simulated according to the Raman-Brillouin quantum model for fixed $\delta^{\prime}=-7$ and $\gamma^{\prime}=1.2$. For each thickness $L_{z}$, the scattered intensity has been separated into diagonal $\left(I_{\text {on }}\right)$ and off-diagonal $\left(I_{\text {off }}\right)$ contributions. When it is too weak $I_{\text {off }}$ has been multiplied by the indicated rescaling factor. Calculation were performed for a limited number of low-frequency confined modes. The inset shows the evolution of $I_{\text {on }}, I_{\text {off }}$ and $I_{\text {on }} / I_{\text {off }}$ as a function of layer thickness. For these plots the intensities were integrated over the whole spectrum.

the excited electronic state $e$ can be different from the one, $e^{\prime}$, giving rise to the optical emission of the scattered photon. The off-diagonal contribution to the Raman-Brillouin spectra becomes dominant for $k_{i(s)} L_{z} \gg 1$ (plots for $L_{z}=100 \mathrm{~nm}$ in Fig. 6). For infinite systems the diagonal contribution is forbidden whereas the off-diagonal contribution gives a single scattered peak: a Brillouin peak located at the acoustic vibration frequency of the wave vector $k_{m}=k_{i}-k_{s}$.

The inset of Fig. 6 shows the evolution of the diagonal $\left(I_{\text {on }}\right)$ and off-diagonal $\left(I_{\text {off }}\right)$ Raman-Brillouin intensities versus layer thickness. These curves are particularly important for analyzing experimental data. Indeed, since the intensities ratios between the Raman-Brillouin lines, due to confined acoustic vibrations, strongly depend on the spatial distribution of the electronic density it is important to evaluate, for a given layer thickness, the relative contributions of diagonal and off-diagonal RBED as both do not have the same spatial distribution (Fig. 5). From the evolution of $I_{\text {on }}$ and $I_{\text {off }}$ one can see that for $\left(L_{z} \lesssim 30 \mathrm{~nm}\right)$ the contribution of the offdiagonal RBED to the Raman-Brillouin scattering can be neglected.

Notice that for $L_{z}=25 \mathrm{~nm}$ and $100 \mathrm{~nm}$ the anti-Stokes intensity is larger than the Stokes intensity because of the stronger outgoing resonance for the anti-Stokes scattering. As a matter of fact, $\delta^{\prime}=-7$ and $\gamma^{\prime}=1.2$ correspond to detun- 


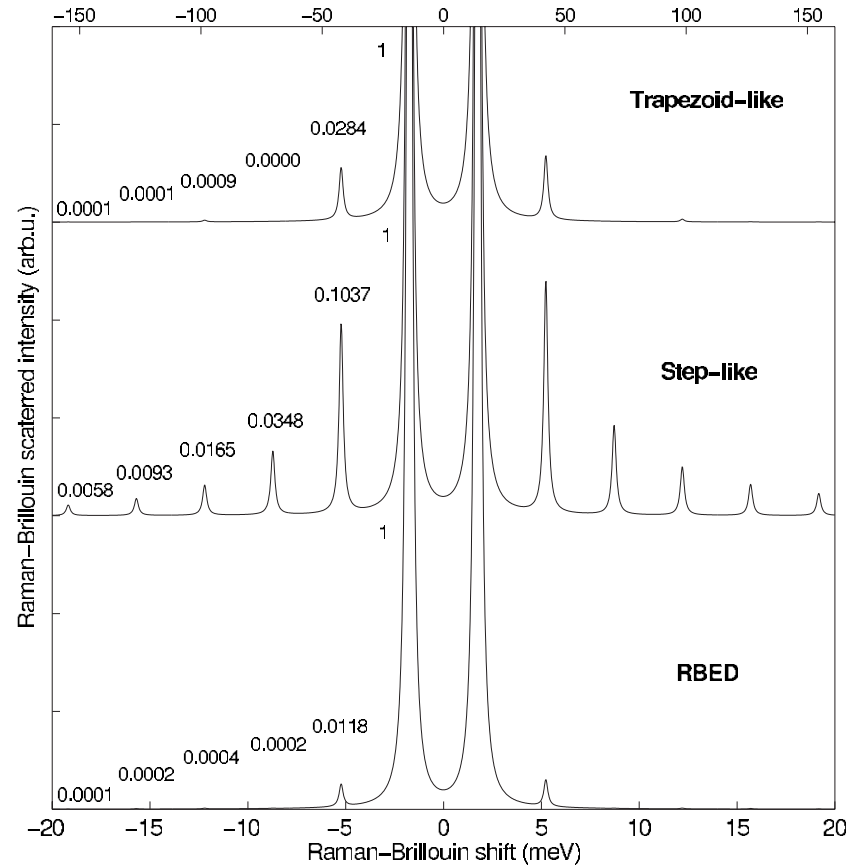

FIG. 7. Inelastic light scattering spectra simulated according to the Raman-Brillouin quantum model $\left(\delta^{\prime}=-7\right.$ and $\left.\gamma^{\prime}=1.2\right)$ and using the photoelastic model with steplike and trapezoidlike profiles of the photoelastic coefficient. The layer thickness is $10 \mathrm{~nm}$. Each spectrum has been normalized to the intensity of the first lowfrequency peak (out of the vertical scale). The intensities ratios, with respect to the first peak, are indicated for the anti-Stokes scattering.

ing $\delta=-1.25 \mathrm{meV}$ and homogeneous broadening $\gamma$ $=0.25 \mathrm{meV}$ for $L_{z}=100 \mathrm{~nm}$. These values indicate that the energy separation between the Stokes and anti-Stokes frequencies is comparable to the detuning and smaller than the homogeneous broadening. In that case, strong differences between outgoing resonances in the Stokes and anti-Stokes regions are indeed expected.

\section{Steplike, trapezoidlike, and RBED profiles}

Figure 7 shows the spectra calculated in the frame of the Raman-Brillouin quantum model and of the photoelastic model for a layer thickness $L_{z}=10 \mathrm{~nm}$. The trapezoidlike photoelastic coefficient used in the PE model is the one that fits the RBED for $L_{z}=10 \mathrm{~nm}$ (see Fig. 4). Each spectrum was normalized to the intensity of the first low-frequency peak. Some differences in the peak intensities ratios can be noticed. These differences lie in the spatial distribution of the $\mathrm{RBED}$ and of the photoelastic coefficient profiles.

As indicated in Fig. 7, the intensities ratios of the peaks generated with the PE model using either the steplike or the trapezoidlike profile are different. The wavelengths of the lowest frequency peaks associated with the $m=1$ and 3 confined modes [see Eq. (3)] are comparable to the layer thickness. They are less sensitive to the details of the photoelastic coefficient profile than the vibration modes at higher frequencies. Indeed, the Raman-Brillouin intensities of the high frequency peaks due to the $m=5,7,9$, and 11 modes are overestimated by the steplike PE profile.

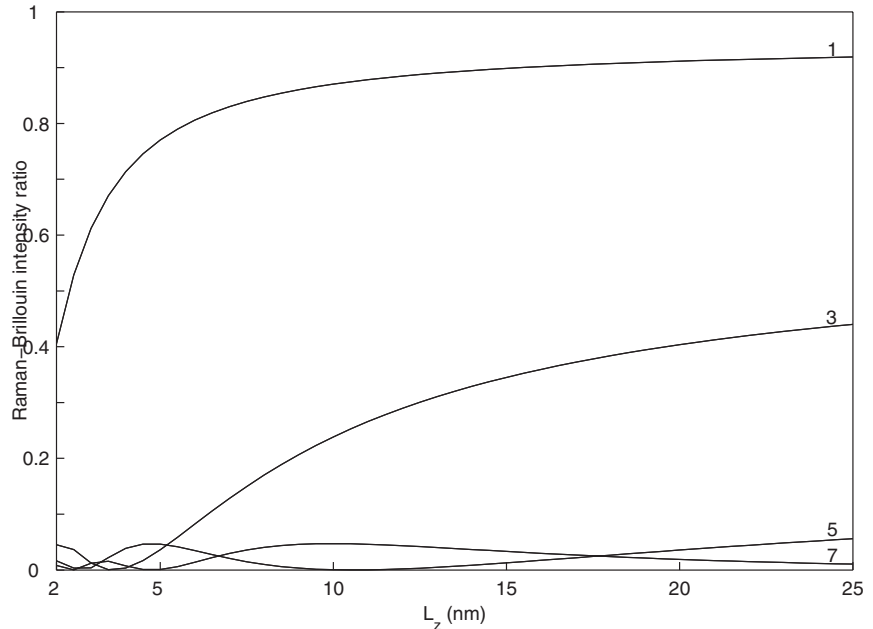

FIG. 8. $I_{\text {trap }} / I_{\text {step }}$ Raman-Brillouin intensities ratios, versus layer thickness, calculated using the PE model with either the trapezoidlike or the steplike PE profiles. The trapezoid profiles are those which fit the RBED for excitation energy $E_{i}=4.075 \mathrm{eV}$ and homogeneous broadening $\gamma=25 \mathrm{meV}$. Results are shown for the $m=1,3$, 5 , and 7 confined vibration modes.

In Fig. 8 are plotted the trapezoidlike/steplike RamanBrillouin intensities ratios as a function of layer thickness $L_{z}$. These plots were generated using the analytical expression

$$
I_{\text {trap }} / I_{\text {step }}=\left(\frac{\sin \left(k_{m} z_{-}\right)}{k_{m} z_{-}}\right)^{2}
$$

which is a simple squared sinc function, where $k_{m}=m \pi / L_{z}$; the trapezoidlike profiles (characterized by $z_{-}$) are those discussed in the preceding section (and plotted in Fig. 4). These profiles were obtained for a fixed excitation energy (4.075 eV) and homogeneous broadening (25 meV), and for layer thickness ranging from 2 to $25 \mathrm{~nm}$ (plotted range in Fig. 8). As one can see, the convergence between the trapezoidlike and steplike models is rather rapid for the first confined mode $m=1$ : the intensity ratio is around 0.4 for $L_{z}$ $=2 \mathrm{~nm}$ and reaches 0.9 for $L_{z}=17 \mathrm{~nm}$. For the $m=3$ mode this ratio is only 0.33 at $L_{z}=17 \mathrm{~nm}$ and less than 0.05 for the $m=5$ and 7 modes. The shorter the wavelength (in comparison with $z_{-}$) the greater the sensitivity to the PE profile.

In most of the published works, ${ }^{4,11,12}$ the photoelastic model, with the crude assumption of a steplike photoelastic coefficient, succeeded in simulating the acoustic phonons induced Raman scattering and the modulation of the optical response observed in time-resolved pump-probe experiments. ${ }^{15-18}$ However, in some cases, for instance in short period superlattices $^{38}$ (i.e., with layer thicknesses smaller that the acoustic wavelengths), or for excitation close to optical resonances, ${ }^{38,39}$ strong deviations between measured and calculated Raman intensities are noticed. In these situations the PE profile can strongly deviate from a steplike profile as shown in Figs. 1, 3, and 4. Therefore, we propose to improve the $\mathrm{PE}$ model (in the high vibration frequency range) by using trapezoidlike, instead of steplike, profiles for the photoelastic coefficient. The dependence of these profiles on the 
layer thickness has been discussed in the previous section. Nevertheless, one must keep in mind that these profiles depend on the excitation energy.

Moreover, experimental data were reported in Ref. 13 on silicon membranes with thicknesses ranging from 24 to $32 \mathrm{~nm}$. The light scattering was excited below the fundamental direct transition. It has been shown that the PE model with a steplike photoelastic constant, well accounts for the Raman-Brillouin intensities associated to the first confined acoustic vibrations. However, some deviations of the simulated spectra from the measured ones can be noticed for the high frequency confined vibrations. According to Fig. 4, differences between the Raman-Brillouin intensities calculated with the trapezoidlike and steplike profiles are indeed expected for such thin layers. It is worthwhile to mention that due to the low scattering efficiency of very thin layers, experiments are usually performed close to resonance with some optical transitions involving confined electronic states. This enhances the Raman-Brillouin scattering but the latter can no longer be described in the frame of the PEM whatever the profile of the photoelastic constant (trapezoid or steplike). As shown in Fig. 1 the RBQM is more appropriate in the case of resonant excitation.

It is worthwhile to underline that the experimental Raman-Brillouin peak intensities strongly depend on the incident and scattered photon fields, ${ }^{13}$ i.e., on the scattering configuration (forward and backward scattering). In our calculations we assumed simple standing electromagnetic waves (cosine functions). More complete modelling of the optical properties is required to compare directly with experiments.

The scattered intensities generated with the RBQM differ from those obtained with the PEM for both the steplike and trapezoidlike profiles. Although, the latter is based on a good approximation of the RBED distribution inside the layer, it does not take into account explicitly outgoing resonance effects. Indeed, the RBED depends on the excitation energy, optical transitions (energy, homogeneous broadening) and also on the scattered photon energy, i.e., on the vibration mode energy [resonance factor for the outgoing photons in Eq. (6)]. This was not taken into account while fitting the RBED with trapezoidlike profiles. In other words, we assumed vibration energies smaller than the homogeneous broadening of the electronic transitions and thus identical resonance factors for the incident and scattered photons. One can see in Fig. 7 that the explored vibration energy range (40 meV in both Stokes and anti-Stokes regions) is larger than the homogeneous broadening $(25 \mathrm{meV})$ of the optical transitions. So, strictly speaking, for a given layer thickness and excitation energy, one should determine a trapezoidlike profile for each scattered photon energy (i.e., for each emitted or absorbed vibration mode). Of course, this points out the limits of the PE model even if size-dependent trapezoidlike profiles are included.

\section{CONCLUSION}

In summary, we have introduced an effective electronic density in the Raman-Brillouin quantum model which allows one to discuss, in a direct and a rather simple way, resonance effects and size dependence of the spectra even when a large number of intermediate electronic states are involved in the light scattering. In particular, we have pointed out the importance of diagonal electron-vibration matrix elements for nanosized objects, as they are responsible for the main contribution to the Raman-Brillouin scattering. The effective Raman Brillouin electronic density also serves to investigate the validity of the steplike profile of the photoelastic coefficient usually assumed in the photoelastic model: we have shown that for layer thickness smaller than $25 \mathrm{~nm}$ the trapezoidlike profile is more realistic than the steplike profile. The Raman-Brillouin spectra calculated using both profiles are comparable for the lowest frequency vibration mode $(\mathrm{m}$ $=1$ confined mode) and for layer thickness larger than $17 \mathrm{~nm}$. The profile of the photoelastic coefficient has strong impacts on the Raman-Brillouin intensities of the highest frequency confined modes $(m=3,5,7, \ldots)$, particularly for very thin layers (below $10 \mathrm{~nm}$ ). As a matter of fact, we found that the intensities of the $m=5,7,9, \ldots$ modes are overestimated by a steplike profile: no convergence between the intensities calculated with the steplike and trapezoidlike profiles was observed for layer thickness up to $25 \mathrm{~nm}$. Moreover, we have shown that, due to optical selection of confined electronic states, neither the steplike nor the trapezoidlike profiles are able to approximate the RamanBrillouin electronic density for resonant excitation. The concepts discussed in this work are quite general and can be extended to analyze the Raman-Brillouin scattering in a variety of low-dimensional systems.
*Present address: GES-UMR 5650 Université Montpellier 2/CNRS.

${ }^{1}$ J. L. Merz, A. S. Barker, and A. C. Gossard, Appl. Phys. Lett. 31, 117 (1977).

${ }^{2}$ C. Colvard, R. Merlin, M. V. Klein, and A. C. Gossard, Phys. Rev. Lett. 45, 298 (1980).

${ }^{3}$ M. V. Klein, IEEE QE-22, 1760 (1986).

${ }^{4}$ B. Jusserand and M. Cardona, Light Scattering in Solids V: Superlattices and Other Microstructures (Springer, Berlin, 1989).

${ }^{5}$ P. Y. Yu and M. Cardona, Fundamentals of Semiconductors:
Physics and Material Properties (Springer, Berlin, 1996).

${ }^{6}$ T. Ruf, Phonon Raman Scattering in Semiconductors, Quantum Wells and Superlattices (Springer, Berlin, 1998).

${ }^{7}$ A. Mlayah and J. Groenen, Light Scattering in Solids IX: Resonant Raman Scattering by Acoustic Phonons in Quantum Dots (Springer, Berlin, 2006), p. 230.

${ }^{8}$ D. F. Nelson and M. Lax, Phys. Rev. B 3, 2778 (1970).

${ }^{9}$ F. Pockels, Ann. Phys. 11, 726 (1903).

${ }^{10}$ O. Lazarenkova and A. Balandin, Nanotechnology 3, 526 (2003).

${ }^{11}$ M. Giehler, T. Ruf, M. Cardona, and K. Ploog, Phys. Rev. B 55, 
7124 (1997).

${ }^{12}$ A. Fainstein and B. Jusserand, Semicond. Sci. Technol. 18, 5377 (2003).

${ }^{13}$ C. M. Sotomayor-Torres, A. Zwick, F. Poinsotte, J. Groenen, M. Prunilla, J. Ahopelto, A. Mlayah, and V. Paillard, Prog. Solid State Chem. 1, 2609 (2004).

${ }^{14}$ A. Fainstein and B. Jusserand, Light Scattering in Solids IX: Raman Scattering in Resonant Cavities (Springer, Berlin, 2006), p. 15.

${ }^{15}$ C. Thomsen, H. T. Grahn, H. J. Maris, and J. Tauc, Phys. Rev. B 34, 4129 (1986).

${ }^{16}$ O. Matsuda and O. Wrigth, Rev. Sci. Instrum. 74, 895 (2003).

${ }^{17}$ B. Perrin, C. Rossignol, B. Bonello, and J. Jeannet, Physica B 263-264, 571 (1999).

${ }^{18}$ A. Devos, J. F. Robillard, R. Cote, and P. Emery, Phys. Rev. B 74, 064114 (2006).

${ }^{19}$ D. Kop'ev, D. Mirlin, V. Sapega, and A. Sirenko, JETP Lett. 51, 708 (1990).

${ }^{20}$ V. F. Sapega, V. I. Belitsky, A. J. Shields, T. Ruf, M. Cardona, and K. Ploog, Solid State Commun. 84, 1039 (1992).

${ }^{21}$ G. Bachelier, A. Mlayah, M. Cazayous, J. Groenen, A. Zwick, H. Carrere, E. Bedel-Pereira, A. Arnoult, A. Rocher, and A. Ponchet, Phys. Rev. B 67, 205325 (2003).

${ }^{22}$ A. A. Sirenko, V. I. Belitsky, T. Ruf, M. Cardona, A. I. Ekimov, and C. Trallero-Giner, Phys. Rev. B 58, 2077 (1998).

${ }^{23}$ S. Gupalov and I. Merkulov, Phys. Solid State 41, 1349 (1999).

${ }^{24}$ J. R. Huntzinger, J. Groenen, M. Cazayous, A. Mlayah, N. Bertru, C. Paranthoen, O. Dehaese, H. Carrère, E. Bedel, and G. Armelles, Phys. Rev. B 61, R10547 (2000).
${ }^{25}$ M. Cazayous, J. R. Huntzinger, J. Groenen, A. Mlayah, S. Christiansen, H. P. Strunk, O. G. Schmidt, and K. Eberl, Phys. Rev. B 62, 7243 (2000).

${ }^{26}$ M. Cazayous, J. Groenen, J. R. Huntzinger, A. Mlayah, and O. G. Schmidt, Phys. Rev. B 64, 033306 (2001).

${ }^{27}$ M. Cazayous, J. Groenen, A. Zwick, A. Mlayah, R. Carles, J. L. Bischoff, and D. Dentel, Phys. Rev. B 66, 195320 (2002).

${ }^{28}$ A. G. Milekhin, A. I. Nikiforov, O. G. Pchelyakov, S. Schulze, and D. R. T. Zahn, JETP Lett. 73, 461 (2001).

${ }^{29}$ A. G. Milekhin, A. I. Nikiforov, O. G. Pchelyakov, S. Schulze, and D. R. T. Zahn, Nanotechnology 13, 55 (2002).

${ }^{30}$ A. G. Milekhin, A. I. Nikiforov, O. P. Pchelyakov, S. Schulze, and D. R. T. Zahn, Physica E (Amsterdam) 13, 582 (2002).

${ }^{31}$ J. R. Huntzinger, A. Mlayah, V. Paillard, A. Wellner, N. Combe, and C. Bonafos, Phys. Rev. B 74, 115308 (2006).

${ }^{32}$ M. Yamanishi, N. Mikoshiba, and T. Minami, Phys. Rev. B 21, 4774 (1980).

${ }^{33}$ J. He, B. Djafari-Rouhani, and J. Sapriel, Phys. Rev. B 37, 4086 (1988).

${ }^{34}$ M. A. Green, J. Appl. Phys. 67, 2944 (1990).

${ }^{35} \mathrm{~J}$. Singh, Physics of Semiconductors and Their Heterostructures (McGraw-Hill, New York, 1993).

${ }^{36}$ M. Cardona and F. H. Pollak, Phys. Rev. 142, 530 (1966).

${ }^{37}$ J. C. Phillips, Phys. Rev. 125, 1931 (1962).

${ }^{38}$ R. W. G. Syme, D. J. Lockwood, and J. M. Baribeau, Phys. Rev. B 59, 2207 (1999).

${ }^{39}$ J. Sapriel, J. He, B. Djafari-Rouhani, R. Azoulay, and F. Mollot, Phys. Rev. B 37, 4099 (1988). 